\title{
Detecting IoT Devices and How They Put Large Heterogeneous Networks at Security Risk
}

\author{
Sharad Agarwal ${ }^{1,2, *(1)}$, Pascal Oser ${ }^{3,4}\left(\mathbb{C}\right.$ and Stefan Lueders ${ }^{3}$ (1) \\ 1 CMS Experiment, European Organization for Nuclear Research (CERN), 1211 Geneva, Switzerland \\ 2 Department of Physics, University of Wisconsin Madison, Madison, WI 53706, USA \\ 3 CERN Computer Security Team, European Organization for Nuclear Research (CERN), \\ 1211 Geneva, Switzerland; p.oser@cern.ch (P.O.); Stefan.Lueders@cern.ch (S.L.) \\ 4 Institute of Distributed Systems, Ulm University, Helmholtzstraße 16, 89081 Ulm, Germany \\ * Correspondence: sharad.agarwal@cern.ch; Tel.: +33-769-465-489
}

Received: 14 August 2019; Accepted: 19 September 2019; Published: 23 September 2019

\begin{abstract}
The introduction of the Internet of Things (IoT), i.e., the interconnection of embedded devices over the Internet, has changed the world we live in from the way we measure, make calls, print information and even the way we get energy in our offices or homes. The convenience of IoT products, like closed circuit television (CCTV) cameras, internet protocol (IP) phones, and oscilloscopes, is overwhelming for end users. In parallel, however, security issues have emerged and it is essential for infrastructure providers to assess the associated security risks. In this paper, we propose a novel method to detect IoT devices and identify the manufacturer, device model, and the firmware version currently running on the device using the page source from the web user interface. We performed automatic scans of the large-scale network at the European Organization for Nuclear Research (CERN) to evaluate our approach. Our tools identified 233 IoT devices that fell into eleven distinct device categories and included 49 device models manufactured by 26 vendors from across the world.
\end{abstract}

Keywords: Internet of Things; security; vulnerabilities and protective measures; control network security; operation in multi-user environments; risk assessment

\section{Introduction}

The Internet of Things has become the latest trend in today's world. For 2020, the installed base of Internet of Things devices is forecast to grow to almost 31 billion worldwide [1]. Nowadays, devices like printers, switches, routers, phones, and any other electrical devices are all interconnected to increase the ease of access and maintenance, but at the same time it increases the security risk of being compromised.

IoT devices do not have the traditional host-centric security solutions like antiviruses, firewalls, or any safety feature to detect malware. Instead, they run on certain firmware that is hardware-specific, and each type of device has a different protocol on whose principles it runs. As the IoT devices collect a lot of data, these firmwares should be developed by the manufacturers in a secured style, but is rarely the case. Access to the data collected and stored by these devices can aid criminals to gain a lot of sensitive information, like patients' healthcare data or video footage of the cameras.

The European Organization for Nuclear Research (CERN), the world's largest High Energy Physics Laboratory and home to the Large Hadron Collider (LHC), is running a plethora of embedded IoT devices. The users in CERN are from a wide variety of fields, ranging from physicists to all kinds of engineers. CERN hosts four major physics experiments-Compact Muon Solenoid (CMS), Atlas, Alice, and Large Hadron Collider beauty (LHCb)—and many other small experiments, which employ 
a wide range of IoT devices. To name a few, there are programmable logic controllers (PLCs), arduinos, oscilloscopes, thermometers, cameras, and others that are extensively used to run the experiments and the LHC successfully. Like all other international organizations, CERN also operates a large technical infrastructure consisting of other general purpose IoT devices such as CCTV cameras, printers, and IP phones. It is important to know the security footprint of the IoT hardware that is integrated into its network complex: unknown devices can run on firmware versions that are not updated and use old legacy code, which introduces vulnerabilities. However, to secure a device, we need to first learn all about the device. With CERN as our primary resource, we argue that insecure IoT devices can escalate the security risk inherent in large heterogeneous networks.

CERN provides easy network access and allows users to set up and register devices that might help them in their work. Users set up their devices on the CERN network by only registering the media access control (MAC) address of their devices. As the network database does not provide sufficient information to identify and differentiate different IoT devices running on the network, the first step we took was to identify the devices installed at CERN and then did a manual security assessment. As shown in Figure 1b, we classify the identified IoT devices into four vulnerability classes and adapt this paradigm to the CERN network. As IoT devices are networked, they are attractive targets and may become the weakest link for breaking into a secure infrastructure [2], or instead leak sensitive information [3] about users and their behaviors [4]. Integrating these unsecured IoT devices in mission-critical networks with industrial control systems, may put directly controlled assets at risk and possibly endanger the whole connected facility. Earlier this year, the European Union Agency for Network and Information Security (ENISA) published guidelines for the development or repositioning of standards, facilitating the adoption of standards and governance of European Union (EU) standardization in the area of Network Information Security (NIS) [5], but the manufacturers, consumers, and the EU Authorities have not yet fully implemented it.

\section{The current basis is 900 loT devices, detected to be connected to CERN's General Purpose Network}

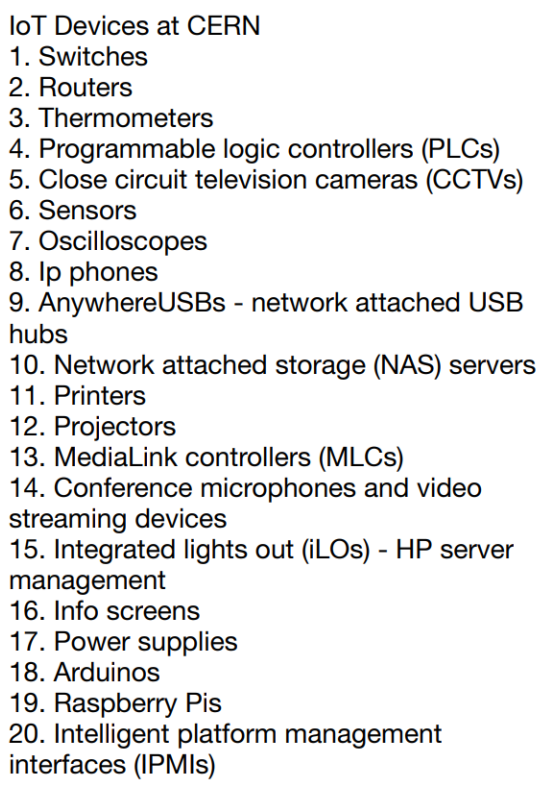

(a)

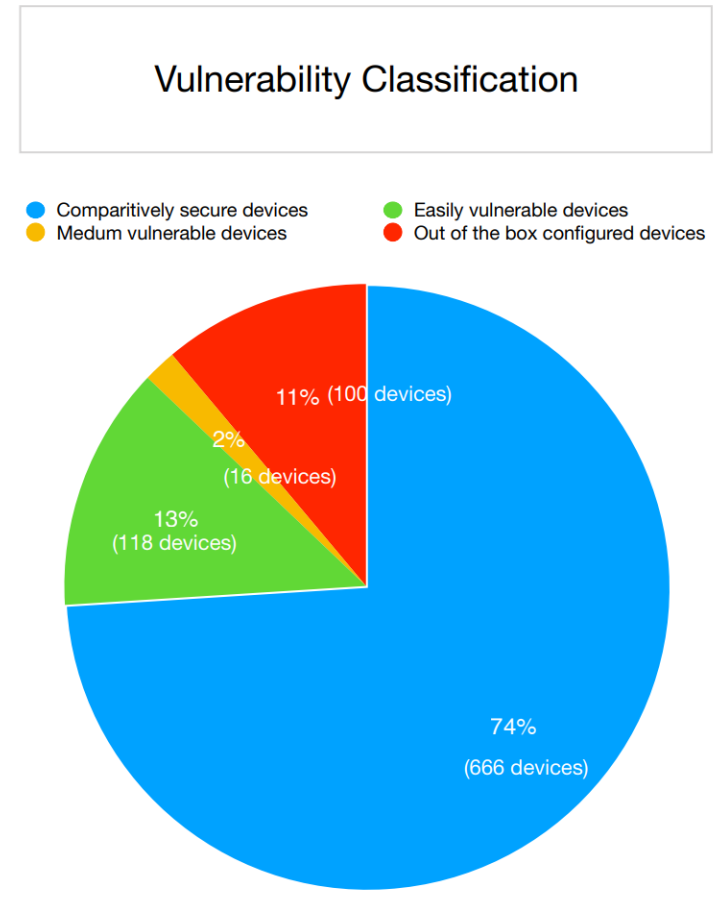

(b)

Figure 1. (a) Overview of Internet of Things (IoT) devices at the European Organization for Nuclear Research (CERN). (b) Vulnerability classification of Internet of Things(IoT) devices at the European Organization for Nuclear Research (CERN). 
In this paper, we identify and present a security assessment of 20 categories of various devices connected to the CERN network, as shown in Figure 1. These 20 categories, mentioned in Figure 1a, were identified by us using our "NetScanIoT" tool. The vulnerability classification in Figure $1 \mathrm{~b}$ was also done by us as per our Vulnerability Assessment results, discussed later in the paper. We not only detected unprotected ports that allow changing the device's configuration, but also the devices that are prone to remote code execution. Remote code execution can be used as a gateway for an attacker to gain access to the internal network from the outside and dig further while operating on a trustworthy device.

\subsection{Contributions}

In this paper, we present an approach to scan the large heterogeneous network without causing faults on remote devices and identify IoT device models based on the web interface. Installing or modifying anything on the device under test (DUT) is not needed. We list our contributions as follows.

- We developed two tools: "NetScanIoT" tool and "Web-IoT Detection (WID)" tool;

The "NetSanIoT" tool detects IoT devices on a large heterogeneous network and is able to detect 20 categories of IoT devices;

The "Web-IoT Detection (WID)" tool identifies the manufacturer name, model, and firmware versions of the respective IoT device. It is able to identify $92.45 \%$ of IoT device models and $100 \%$ of IoT device that have a web user interface;

- We implemented a manual security assessment of 20 categories of devices that were identified by our "NetScanIoT" tool on the highly heterogeneous, large-scale network at CERN.

None of the devices installed at CERN are CERN-specific or manufactured only for CERN. The devices installed are manufactured by various vendors from across the world, readily available and also used by other organizations and individuals. The next subsection introduces related work and Section 2 explains the methodologies used for detection of IoT devices and the vulnerability assessment. Section 3 describes the evaluation of the "Web-IoT Detection" (WID) approach following the results of the vulnerability assessment, before we discuss our findings.

\subsection{Related Work}

Most of the related work has identified very few unique categories of IoT devices by scanning a network. Scanning can be done either actively or passively. Active scanning is one-to-one probing communication and passive is where the client listens to every channel's transmission, which is monitored periodically. Some tools also employ web interface fingerprinting but have assumptions and constraints like working on only single-page applications or analyzing the Hyper Text Transfer Protocol (HTTP) response messages only [6]. Other tools depend on Nmap [7] port scanning and downloading the landing page using Curl [8] to find the firmware version for the IoT devices [9], which does not work for all IoT devices. An IP-based IoT Device Detection approach requires the knowledge of servers run by the manufacturers and are able to evaluate using only ten device models by seven vendors [10]. Another solution, "IoTScanner", detects, by passive measurement, the identifying devices using the packet's MAC address [11]. We cannot use passive scanning in our tool as we have 1000 s of star points at CERN and depending only on MAC address is not sufficiently reliable.

\section{Materials and Methods}

This section introduces the different approaches we developed to detect and identify IoT devices and the vulnerability assessment performed by us. The first subsection explains the tools we developed and the next subsection tells about the vulnerability assessment we tried manually on these identified IoT devices. 


\subsection{Identification}

Although all devices connected to CERN's networks need to be registered, CERN does not have a specific database for IoT devices in particular. There are hundreds of devices running on various networks and new devices being installed every day. CERN provides a way for all users and visitors to add their devices to the network by just registering the MAC address of the device. The central network database of CERN does not have any other detailed information. Therefore, there is no way to distinguish an IoT device from a computer or a cellphone. As we cannot depend solely on MAC addresses to identify an IoT device, we developed tools to detect and identify these IoT devices. These tools provide more information about the device, which can help the administrators in maintaining the security of these devices. This subsection explains the tools we developed to solve this problem as follows.

\subsubsection{NetScanIoT Tool}

We wrote a Python [12] tool called NetScanIoT, which pings the devices within the network and checks the ICMP [13] message if the target is reachable. If the device response is positive, we go for a nslookup [14] to find the hostname and save the list of the IP addresses along with their hostnames. We prefiltered the output devices by port scanning and then manually also removed the non-IoT devices from the list connected to the network. We were able to identify 20 categories of IoT devices, as shown in Figure 1a. Figure 2 shows a graphical working of the NetScanIot tool. With the help of this tool, we were able to detect 900 physical IoT devices.

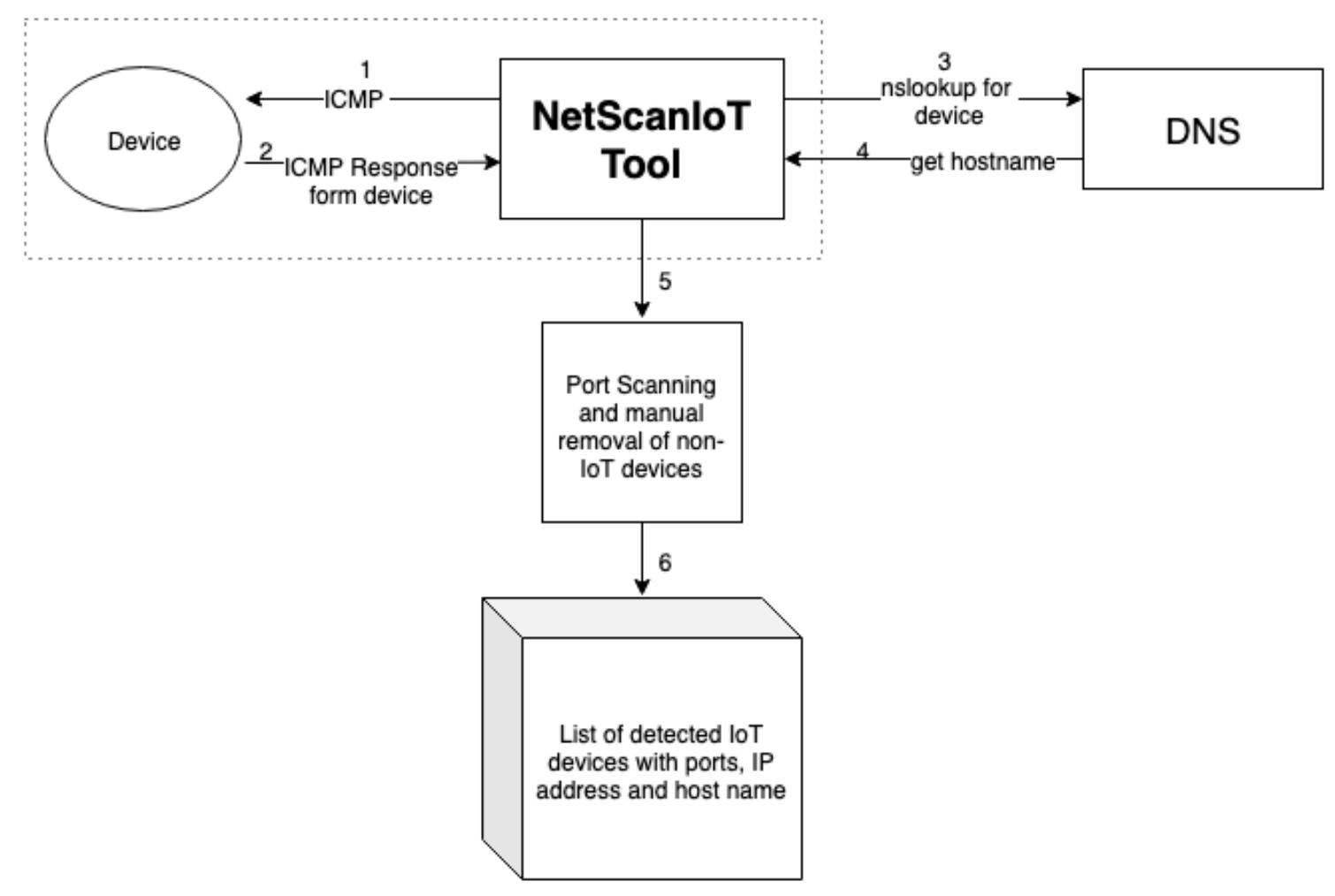

Figure 2. Overview of NetScanIoT tool.

\subsubsection{Web-IoT Detection (WID) Tool}

Scraping a web page can be done with many available tools these days, but with so many different manufacturers, the challenge becomes tough. We initially tried to use Wget [15], Curl, Scrapy [16], and other tools, but there are multiple web pages that require to render JavaScript code, which these tools can not. The reason for doing so is that $20 \%$ of the device models' web pages render JavaScript first 
to show the complete page source. Therefore, we wrote the WID tool in Python using Selenium [17] in headless mode, which renders the web page with a web driver (Chrome/Firefox) to get the page source. We first analyzed the page source manually and identified six classifiers. With the help of Beautiful Soup [18], we used these classifiers and automated the process to find the category of the IoT device. WID not only scrapes the index page of the device, but also scrapes the sub-URLs recursively, to identify the devices' information such as the model and the firmware version running on the device.

Figure 3 shows an overview of the Web-IoT Detection tool and explains the working of the tool to identify the IoT device. With the help of this tool, we were able to identify 233 physical IoT devices, which have a web interface. Figure 4 shows a sample working of the WID tool that takes an IP address as input and identifies the device, model, and firmware version. We also present the classifiers in the example, which are analyzed from different sub-URLs that the tool scraped.

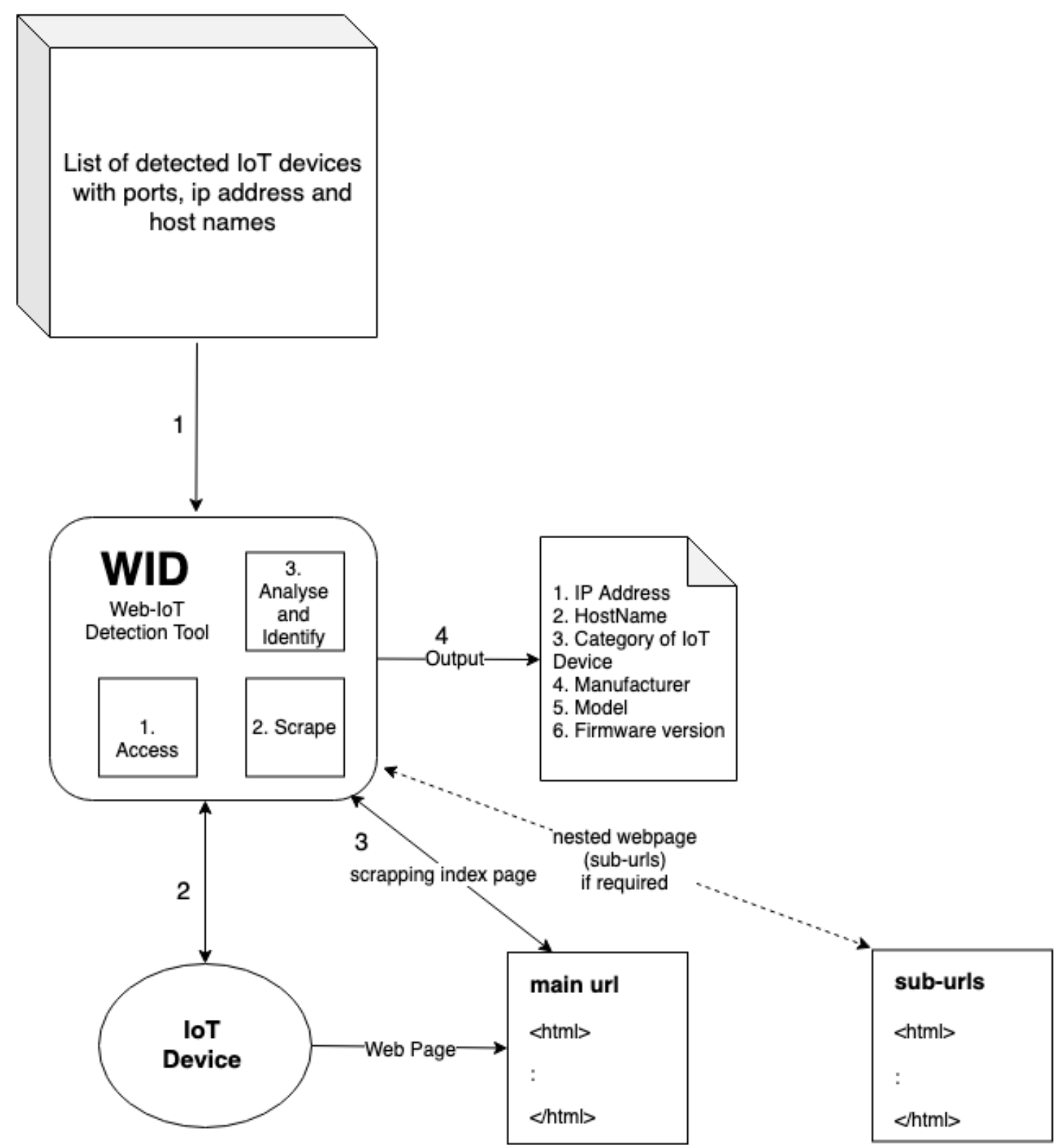

Figure 3. Overview of Web-IoT detection (WID) tool. 


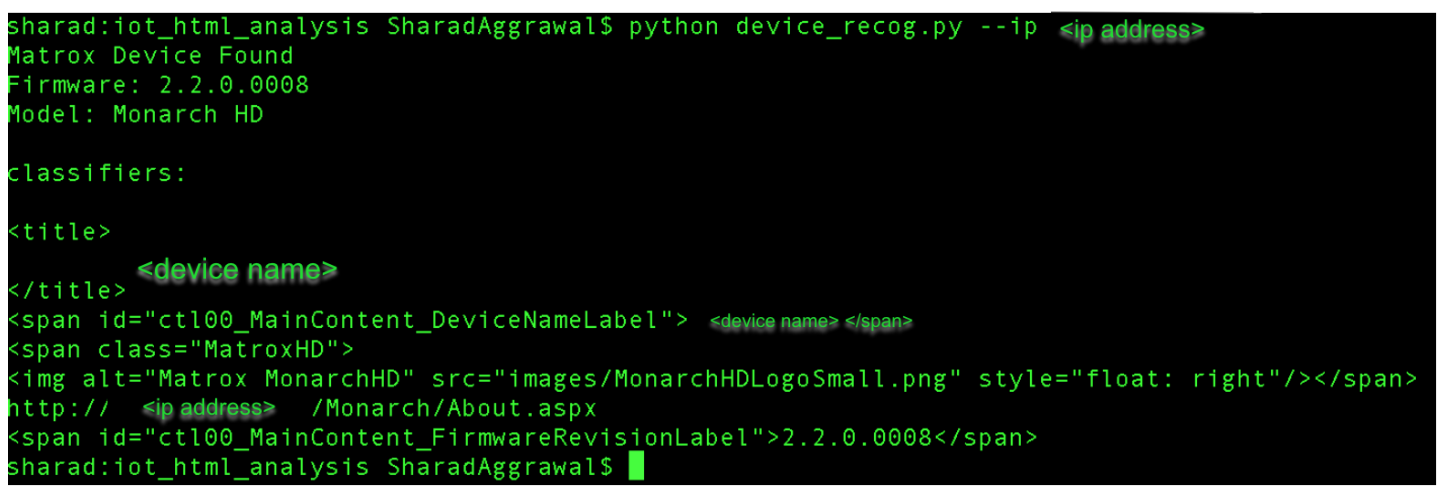

Figure 4. Sample working of Web-IoT detection (WID) tool.

The WID tool produces the following output.

- IP-address and the host name of the device;

- Web page availability of the device;

- Category of device identified;

- Manufacturer/Vendor name;

- Model name;

- Firmware version.

\subsection{Vulnerability Assessment}

As the Internet of Things is a very new technology, there is no specially designed vulnerability assessment tool that is known to us. There are some general tools like Nessus [19], Open vulnerability assessment system (OpenVAS) [20], and others, but these do not deliver good results, as they do for regular clients or servers. So, we started with the network and web interface of all the IoT devices since it is the primary interface through which the users can connect to the devices. We started looking for web interface injections and attacks to check for the devices. The first step was to use the top Open web application security project (OWASP) IoT Vulnerabilities [21] for investigating the IoT device's web interface and tried to get administrative access. Our next aim was to find the network side vulnerabilities by scanning the devices and checking the open and filtered ports vulnerable to attack—some of them being secure shell (SSH) [22], Telnet [23], Session initiation protocol (SIP) [24], Real time streaming protocol (RTSP) [25], and JetDirect [26]—and get administrative access into the configurations of the devices. We used software like Printer exploitation toolkit (PRET) [27] and Routerscan [28]. We also modified three available exploits from the Google Hacking Database [29] to find vulnerable IoT devices on the network.

\section{Results}

\subsection{Evaluation of Tools}

In this section, we elaborate on the results of the Web-IoT Detection (WID) tool. As mentioned in Table 1, we show the models and manufacturers names that we are successfully able to identify with our tool. It shows that eleven out of 20 categories of devices that have a web interface were identified. We achieve an accuracy of $92.45 \%$ as only 49 out of 53 models were identified by the software using the six classifiers. The "NetScanIoT" and the "Web-IoT Detection" tools can be used in any organization or even by an individual to detect, identify, and use the output information to keep their IoT devices secure. 
Table 1. Devices detected by WID.

\begin{tabular}{|c|c|c|c|c|}
\hline Category & Model & Manufacturer & Quantity & City, Country of Manufacturer \\
\hline Matrox & Matrox Monarch HD & Matrox & 26 & Dorval, QC, Canada \\
\hline Matrox & Matrox LCS & Matrox & 2 & Dorval, QC, Canada \\
\hline Telepresence & $\mathrm{C} 20 / 40$ & Cisco & 10 & San Jose, CA, USA \\
\hline Oscilloscope & Tektronix & Tektronix & 3 & Beaverton, OR, USA \\
\hline Oscilloscope & Lecroy & Teledyne Lecroy & 3 & New York, USA \\
\hline IP Phone & Cisco & Cisco & 2 & San Jose, CA, USA \\
\hline IP Phone & FLX & Revolabs & 1 & Sudbury, MA, USA \\
\hline IP Phone & Yealink & Yealink & 1 & Xiamen, China \\
\hline NAS & Diskstation & Synology & 24 & Taipei, Taiwan \\
\hline Printer & Color Laserjet M553 & Hewlett Packard & 5 & Palo Alto, CA, USA \\
\hline Printer & DCP-L & Brothers & 2 & Aichi Prefecture, Japan \\
\hline Printer & HL-5470 & Brothers & 1 & Aichi Prefecture, Japan \\
\hline Printer & HL-3070CW & Brothers & 1 & Aichi Prefecture, Japan \\
\hline Printer & $\mathrm{mfc}-8370 \mathrm{dn}$ & Brothers & 1 & Aichi Prefecture, Japan \\
\hline Printer & Color Laserjet mfp m277 & Hewlett Packard & 3 & Palo Alto, CA, USA \\
\hline Printer & Laserjet cp $1525 \mathrm{~N}$ & Hewlett Packard & 1 & Palo Alto, CA, USA \\
\hline Printer & Color Laserjet $\mathrm{cm} 1312 \mathrm{nfi} \mathrm{mfp}$ & Hewlett Packard & 1 & Palo Alto, CA, USA \\
\hline Printer & Laserjet $400 \mathrm{~m} 401$ & Hewlett Packard & 1 & Palo Alto, CA, USA \\
\hline Printer & Star Asura & Star POS Printing Soln. & 3 & Shizuoka, Japan \\
\hline Printer & HP envy & Hewlett Packard & 3 & Palo Alto, CA, USA \\
\hline Printer & Photosmart plus printer & Hewlett Packard & 2 & Palo Alto, CA, USA \\
\hline CCTV Camera & ip8365eh & Vivotek & 9 & New Taipei City, Taiwan \\
\hline CCTV Camera & Flexidome ip corner $9000 \mathrm{mp}$ & Bosch & 6 & Gerlingen, Germany \\
\hline CCTV Camera & M1114 & Axis & 2 & Lund, Sweden \\
\hline CCTV Camera & q6000-e & Axis & 3 & Lund, Sweden \\
\hline CCTV Camera & P5635-E MKII & Axis & 4 & Lund, Sweden \\
\hline CCTV Camera & Q24 & Mobotix AG & 3 & Winnweiler, Germany \\
\hline CCTV Camera & $\mathrm{M} 24$ & Mobotix AG & 2 & Winnweiler, Germany \\
\hline CCTV Camera & M25 & Mobotix AG & 6 & Winnweiler, Germany \\
\hline CCTV Camera & DCS-910 & D-link & 2 & Taipei, Taiwan \\
\hline CCTV Camera & AW-HE60H & Panasonic & 2 & Osaka Prefecture, Japan \\
\hline CCTV Camera & SNC-RZ50 & Sony & 1 & Tokyo, Japan \\
\hline PLC & Saia & SBC & 10 & Murten, Switzerland \\
\hline Arduino & Arduino Yun/Uno & Arduino & 9 & Somerville, MA, USA \\
\hline IPMI & ILO & Hewlett Packard & 12 & Palo Alto, CA, USA \\
\hline
\end{tabular}

\subsection{Vulnerability Assessment Results}

After the manual security assessment, we found out that 100 of the 900 devices have the default configuration, and we classify this as "Out of the box configured" category. The devices of this category had no authentication setup on the web user interface or the command-line interface. The "Easily vulnerable" category consisted of 118 devices that had easily guessable or standard manufacturer-configured credentials, which made them easily accessible to users within the network. Apart from this, there were certain devices like thermometers which had a hard-coded super admin password that cannot be changed. We also found 16 devices vulnerable to known exploits, which included Real-Time Streaming Protocol (RTSP) Bypass authentication. This exploit affects two manufacturers' Close Circuit Television Cameras (CCTV) with various running firmware versions. There is more than one model prone to this exploit. Using the PRET software and the JetDirect port, 
we were able to access the configuration of printers installed at CERN. Additionally, we also discovered that we are able to change the standard welcome message on most of the printers.

Once the vulnerability assessment was completed, we wanted to mitigate the vulnerabilities by reporting them to the administrators and users of the devices. Sending emails to each and every affected device administrator and user at CERN was a tedious task, so we used a platform called Fast Incidence Response (FIR) [30] modified at CERN according to our needs. FIR is a centralized platform and is used to report devices to their owners and responsible users. We used this to report about the affected devices to the responsible owners and provided them with more information on how to mitigate the issues. By doing this assessment, we raise security awareness at CERN. Adding all devices from the categories, as mentioned in Figure 1b, there were 234 vulnerable devices, which were reported and suggested solutions to mitigate them as that could have caused security issues.

\section{Discussion}

We present our results to identify and assess IoT devices on a large-scale and in a heterogeneous network. The tools developed by us can be used by any organization or an individual, to detect and identify IoT devices. The information provided by the tools can be used to secure their IoT devices. With our NetScanIoT software, a total of 20 categories of IoT devices were identified successfully. After identifying these devices, we performed a manual vulnerability assessment on them. This assessment showed that IoT manufacturers did not secure their devices and, moreover, certain devices, like the thermometers [31], did not even allow the user to change the credentials at all. The Web-IoT detection (WID) tool was able to identify eleven out of 20 categories of IoT devices consisting of 49 various models, manufactured by 26 different vendors from across the world. We also identified the corresponding manufacturer and firmware version for these 49 device models of IoT devices, which can be used for risk identification, associated with these firmware versions. None of these identified devices are CERN-specific or specially manufactured for CERN. They are the same devices that the manufacturers sell in the global market.

One of the significant findings was that 118 devices administered by 90 users were using default passwords and old firmware versions. The administrators did not consider to change them at all as they were not made aware by any kind of prompt that they should change the default password or update to the latest firmware version of the device. Therefore, we propose periodic scans on all networks to detect devices that might be vulnerable.

We showed that the approach is effective on a large-scale network with a larger dataset compared to similar studies. Moreover, no other work was able to classify this amount of heterogeneous IoT device models by using the web interface. For future work, we plan to identify new types of IoT devices that come up together with industrial IoT devices on our accelerator complex testbed.

\section{Conclusions}

The paper provides a novel approach to detect and identify Internet of Things (IoT) devices on a large heterogeneous network. The paper also explains the vulnerability assessment carried out on the large heterogeneous network at CERN and provides results with a vulnerability classification. The "NetScanIoT" tool and the "Web-IoT Detection" (WID) tool can successfully detect and identify IoT devices and also provide more information such as model, manufacturer and firmware version of the device. The tools provide an accuracy of $92.45 \%$ for identification of an IoT device. The tools were successfully able to identify 29 IoT device models manufactured by 26 different vendors from across the world. 
Author Contributions: Conceptualization, S.A. and P.O.; methodology, S.A. and P.O.; software, S.A.; validation, S.A., P.O., and S.L.; formal analysis, S.A.; investigation, S.A.; resources, S.A.; data curation, S.A.; writing-original draft preparation, S.A.; writing-review and editing, S.A., P.O. and S.L.; visualization, S.A.; supervision, P.O. and S.L.; project administration, S.L.

Funding: This research received no external funding.

Acknowledgments: We thank Hannah Short (CERN) for proofreading that greatly improved the manuscript. This research was supported by European Organization for Nuclear Research (CERN) and CERN Openlab. We thank our colleagues from CERN who provided insights and expertise that greatly assisted the research.

Conflicts of Interest: The authors declare no conflict of interest. The funders had no role in the design of the study; in the collection, analyses, or interpretation of data; in the writing of the manuscript; or in the decision to publish the results.

\section{Abbreviations}

The following abbreviations are used in this manuscript.

$\begin{array}{ll}\text { IoT } & \text { Internet of Things } \\ \text { WID } & \text { Web-IoT Detection } \\ \text { CERN } & \text { European Organization for Nuclear Research } \\ \text { LHC } & \text { Large Hadron Collider } \\ \text { DUT } & \text { Device Under Test } \\ \text { PLC } & \text { Programmable Logic Controller } \\ \text { ENISA } & \text { European Union Agency for Network and Information Security } \\ \text { NIS } & \text { Network Information Security } \\ \text { UI } & \text { User Interface } \\ \text { NAS } & \text { Network Attached Storage } \\ \text { MLC } & \text { Media Layer Controller } \\ \text { IP } & \text { Internet Protocol } \\ \text { MAC } & \text { Media Access Control } \\ \text { HTTP } & \text { Hypertext Transfer Protocol } \\ \text { SSH } & \text { Secure Shell } \\ \text { CCTV } & \text { Close Circuit Television } \\ \text { OWASP } & \text { Open Web Application Security Project } \\ \text { SIP } & \text { Session Initiation Protocol } \\ \text { RTSP } & \text { Real-Time Streaming Protocol } \\ \text { URL } & \text { Uniform Resource Locator } \\ \text { LHCb } & \text { Large Hadron Collider beauty } \\ \text { CMS } & \text { Compact Muon Solenoid } \\ & \end{array}$

\section{References}

1. Internet of Things (IoT). Connected Devices Installed Base Worldwide From 2015 to 2025 (in Billions). Available online: https:/ / www.statista.com/statistics /471264/iot-number-of-connected-devicesworldwide (accessed on 20 June 2018).

2. Bruce Schneier. The Internet of Things Is Wildly Insecure-And Often Unpatchable. 2014. Available online: https: / / www.wired.com/2014/01/theres-no-good-way-to-patch-the-internet-of-thingsand-thatsa-huge-problem (accessed on 18 August 2018).

3. Tianlong, Y.; Vyas, S.; Srinivasan, S.; Yuvraj, A.; Chenren, X. Handling a trillion (unfixable) flaws on a billion devices: Rethinking network security for the internet-of-things. In Proceedings of the 14th ACM Workshop on Hot Topics in Networks, Philadelphia, PA, USA, 16-17 November 2015; p. 5.

4. Bruce Schneier. Will Giving the Internet Eyes and Ears Mean the End of Privacy? Available online: https: / / www.theguardian.com/technology/2013/may/16/internet-of-things-privacy-google (accessed on 18 August 2018).

5. IoT Security Standards Gap Analysis. Available online: https://www.enisa.europa.eu/publications/iotsecurity-standards-gap-analysis (accessed on 18 January 2019). 
6. Costin, A.; Zarras, A.; Francillon, A. Towards automated classification of firmware images and identification of embedded devices. In IFIP International Conference on ICT Systems Security and Privacy Protection; Springer: Cham, Switzerland, 2017; pp. 233-247.

7. Lyon, G.F. Nmap Network Scanning: The Official Nmap Project Guide to Network Discovery and Security Scanning; Insecure.Com, LLC: Palo Alto, CA, USA, 2009.

8. Stenberg, D. Everything-Curl; GitBook: Lyon, France, 2017.

9. Zheng, Z.; Webb, A.; Reddy, A.N.; Bettati, R. IoTAegis: A Scalable Framework to Secure the Internet of Things. In Proceedings of the IEEE 2018 27th International Conference on Computer Communication and Networks (ICCCN), Hangzhou, China, 30 July-2 August 2018; pp. 1-9.

10. Guo, H.; Heidemann, J. IP-Based IoT Device Detection. In Proceedings of the ACM 2018 Workshop on IoT Security and Privacy (IoT S\&P'18), Budapest, Hungary, 20 August 2018; pp. 36-42. [CrossRef]

11. Siby, S.; Maiti, R.R.; Tippenhauer, N.O. IoTscanner: Detecting privacy threats in IoT neighborhoods. In Proceedings of the 3rd ACM International Workshop on IoT Privacy, Trust, and Security IoTPTS 17, Abu Dhabi, UAE, 2 April 2017; pp. 23-30.

12. Python Software Foundation. Python Language Reference, Version 2.7. Available online: http://www. python.org (accessed on 7 August 2019).

13. Deering, S. ICMP Router Discovery Messages. Available online: https://tools.ietf.org/html/ $\mathrm{rfc} 1256$ (accessed on 20 September 2018).

14. Toebes, J.; Turner, B.C.; Walker, D.J. Arrangement in a Server for Providing Dynamic Domain Name System Services for Each Received Request. U.S. Patent 7,499,998, 21 April 2005.

15. Free Software Foundation. GNU Wget 1.20. Available online: https://www.gnu.org/software/wget/ (accessed on 7 August 2019).

16. Scarpy Developers. Scrapy 1.7. Available online: https://docs.scrapy.org/en/latest/intro/overview.html (accessed on 7 August 2019).

17. Python Software Foundation. Selenium 3.141.0. Available online: https://pypi.org/project/selenium/ (accessed on 7 August 2019).

18. Richardson, L. Beautiful Soup Documentation. Available online: https://buildmedia.readthedocs.org/ media/pdf/beautiful-soup-4/latest/beautiful-soup-4.pdf (accessed on 20 September 2018).

19. Tenable.Com. Nessus. Available online: https://www.tenable.com/products/nessus/nessus-professional (accessed on 12 March 2018).

20. Openvas.Org. OpenVAS. Available online: http:/ / openvas.org/ (accessed on 16 February 2018).

21. OWASP. OWASP Internet of Things Project. Available online: https://www.owasp.org/index.php/OWASP_ Internet_of_Things_Project\#tab=IoT_Vulnerabilitiessoftware.html (accessed on 7 May 2018).

22. Ylonen, T.; Lonvick, C. The Secure Shell (SSH) Protocol Architecture. Available online: https://tools.ietf. org / html/rfc4251 (accessed on 20 September 2018).

23. Postel, J.; Reynolds, J.K. Telnet Protocol Specification. Available online: https://tools.ietf.org/html/rfc854 (accessed on 20 September 2018).

24. Rosenberg, J.; Schulzrinne, H.; Camarillo, G.; Johnston, A.; Peterson, J.; Sparks, R.; Handley, M.; Schooler, E. SIP: Session Initiation Protocol. Available online: https://tools.ietf.org/html/rfc3261 (accessed on 20 September 2018).

25. Schulzrinne, H.; Rao, A.; Lanphier, R. Real-Time Streaming Protocol (RTSP). Available online: https://tools. ietf.org/html/rfc2326 (accessed on 20 September 2018).

26. The Free Encyclopedia Wikipedia. JetDirect. Available online: https://en.wikipedia.org/wiki/JetDirect (accessed on 13 June 2017).

27. Müller, J. Exploiting Network Printers. Available online: https://www.nds.ruhr-uni-bochum.de/media/ei/ arbeiten/2017/01/13/exploiting-printers.pdf (accessed on 23 July 2017).

28. Router Scan v2.60 Beta by Stas'M. Available online: http:/ / stascorp.com/load/1-1-0-56 (accessed on 10 May 2018).

29. Google Hacking Database. Available online: https://www.exploit-db.com/google-hacking-database/ (accessed on 7 May 2018).

30. CERN CERT. FIR. Available online: https:/ / github.com/CERN-CERT/FIR (accessed on 25 June 2018). 
31. Agarwal, S.; Oser, P.; Short, H.; Lueders, S. Internet of Things Security. Available online: https://doi.org/10. 5281 /zenodo.1035034 (accessed on 11 March 2018).

Sample Availability: The source code sample will be available from the authors Sharad Agarwal and Pascal Oser after the completion of the PhD of Pascal Oser, in 2020.

(C) 2019 by the authors. Licensee MDPI, Basel, Switzerland. This article is an open access article distributed under the terms and conditions of the Creative Commons Attribution (CC BY) license (http://creativecommons.org/licenses/by/4.0/). 